\section{Spasmodic dysphonia like presentation of stiff person syndrome}

Sir,

Stiff person syndrome (SPS), originally known as Stiff-man syndrome, is a rare neurological auto-immune disorder first described by Moesrch and Woltman in $1956{ }^{[1]}$ It is characterized by the stiffness/rigidity of the axial muscles with painful spasms that are fluctuant in nature. ${ }^{[1]}$ These symptoms are exacerbated by emotional distress, sudden movement of limbs, and other related stimuli. Clinical criteria were established by Gordon et al. and later Lorish et al. to accurately diagnose this disorder. ${ }^{[2]}$ Thompson and Dalakas later expanded this criteria to include tightness of the axial muscles, the progression of stiffness to the limbs, painful spontaneous muscle spasms, and an elevation of positive antiglutamic acid decarboxylase (GAD or amphiphysin) antibodies. ${ }^{[3]}$ Our patient was a 50-year-old female bank manager who was diagnosed with hypothyroidism, Vitiligo and vitamin B12 deficiency a few months before her presentation of SPS. In 2007, she developed a loss of her voice over a few days. Her voice was breathy and soft and she had to put a notable amount of effort when speaking. She was thought to have spasmodic dysphonia. However, ENT evaluation with laryngoscopy showed normal vocal cords on two separate occasions. Several years later, accompanying the loss of voice, she started noticing spasms in her neck with a subjective feeling of impaired breathing.
She described these spasms in correlation with pain in her shoulders and a constant contraction of the neck and lower back. She also developed a mild drooping of the left eyelid and occasional subjective difficulty when swallowing. Results from repetitive nerve stimulation and single fiber EMG for Myasthenia Gravis and Eaton Lambert Syndrome were unremarkable on two occasions. A computed tomography (CT) scan of the chest was normal. By this time, she noticed that her overall condition was worsening with an increased fatigue, which finally led to her inability to work. Finally, a differential diagnosis of SPS was entertained as her serological investigations revealed highly elevated anti-GAD antibodies.

SPS has an auto-immune basis and may be accompanied by other auto-immune diseases in addition to elevated levels of anti-GAD antibodies. ${ }^{[1,3]}$ Furthermore, other antibodies such as anti-amphiphysin and anti-gephryin may be elevated in SPS. ${ }^{[4]}$ Decrease in Gamma Amino Butyric Acid (GABA), one of the main inhibitory neurotransmitters in the central nervous system (CNS) regulated by $\mathrm{GAD}$, leads to a heightened stimulation of the muscles by motor neurons. ${ }^{[5]}$ Positive anti-GAD levels are often implicated with strong antagonistic muscle contractions leading to rigidity in the lower limbs - a key feature in SPS. ${ }^{[4]}$

Interestingly, our patient initially presented with spasmodic dysphonia-like symptoms, which made this case a diagnostic challenge. Also, our patient had a clinical picture similar to cranial nerve involvement, as seen by her ptosis of the eye (predominantly on the left side). Much later in the course of the disease, she had painful neck spasms and visible bilateral contractions of the platysma. She also had trouble swallowing. Her ptosis on her left side was a sustained contraction without the weakness on the left side of the face as she was able to open her eye. Ophthalmology and Rheumatology assessments were unremarkable. Although some of her symptoms allude to cranial nerve or ocular muscle involvement, this SPS rarely affects the cranial nerves, ocular or bulbar muscles. What is of particular interest in our case is the initial presentation of spasmodic dysphonia like symptoms that preceded the classic signs of SPS by two and a half years. The loss of voice may have been due to spasms in the neck, from the underlying SPS, which were intermittent or subtle enough to go unnoticed. It was only years later, with the onset of sustained muscle contractions of the neck and lower back and the hyperlordosis, that SPS was suspected to be involved. This was later confirmed with the elevated titer of anti-GAD antibodies. In many cases described by Lorish et al. in 1989, patients 
afflicted with these limitations adopt a hyperlordotic lumbar posture with an extended position of the neck, which persists while lying down. ${ }^{[2]}$ However, with the initial presentation of spasmodic dysphonia and the seemingly absent classic signs of SPS, there was a significant delay in diagnosis and treatment requiring invasive investigations. This case highlights the importance of considering SPS in the differential diagnosis, when no other explanations of spasmodic dysphonia, cranial or bulbar like symptoms could be found.

\section{Abdul Qayyum Rana, Mohamed Sufian Masroor ${ }^{1}$, Beenish Ismail ${ }^{2}$}

Department of Neurology, Parkinson's Clinic of Eastern Toronto and Movement Disorders Center, Toronto, ${ }^{1} \mathrm{Health}$ Science Program, McMaster University, Hamilton, Canada, ${ }^{2}$ Windsor University School of Medicine, Basseterre, Saint Kitts and Nevis

Address for correspondence: Dr. Abdul Qayyum Rana, Parkinson's Clinic of Eastern Toronto and Movement Disorders Center, 1-2060 Ellesmere Road, Toronto - M1H 2V6, Ontario, Canada. E-mail: ranaaq@yahoo.com

\section{References}

1. Blum P, Jankovic J. Stiff-person syndrome: An autoimmune disease. Movement Disorders 1998;6:12-20.

2. Lorish TR, Thorsteinsson G, Howard FM Jr. Stiff-man syndrome updated. Mayo Clinic Proc 1989;64:629-636.

3. Gershanik OS. Stiff-person syndrome. Parkinsonism and Related Disorders 2009;15:S130-4.

4. Murinson BB. Stiff-person syndrome. Neurologist 2004;10:131-37.

5. Goodson B, Martin K, Hunt T. Stiff person syndrome presenting with sudden onset of shortness of breath and difficulty moving the right arm: A case report. J Med Case Rep 2010;4:118.

\begin{tabular}{|l|l|}
\hline \multicolumn{2}{|c|}{ Access this article online } \\
\hline Quick Response Code: & Website: \\
\hline & www.ruralneuropractice.com \\
\cline { 2 - 2 } & \\
\hline & \\
\hline
\end{tabular}

